\title{
The Fine Structure of a Nuclear Envelope Associated Endosymbiont of Paramecium
}

\author{
By R. A. JENKINS \\ Department of Zoology and Physiology, University of Wyoming, Laramie, \\ Wyoming 82070, U.S.A. \\ (Accepted for publication 21 February 1970) \\ SUMMARY \\ Particles of a newly described endosymbiont of Paramecium, here referred \\ to as epsilon, have a fine structure that is essentially identical to that of \\ Gram-negative bacteria. The symbionts occur only within loculi formed \\ by extension of the outer membrane of the nuclear envelopes.
}

\section{INTRODUCTION}

It is difficult to identify the presumed endosymbionts of Paramecium with a particular micro-organism or to determine the nature of the cytoplasmic interaction of the host cell and symbiont. The endosymbiont mu resembles Gram-negative bacteria (Beale \& Jurand, I960, I966). More recently, Jurand \& Preer (1969) established that the symbionts of all stocks known to kill by rapid lysis are flagellated, Gram-negative bacteria. Beale, Jurand \& Preer (1969) reviewed the biology of the better known endosymbionts of Paramecium aurelia and concluded that the symbionts represent a miscellaneous collection of bacteria which are adapted to an intracellular niche. It is generally accepted that the existence and perpetuation of an endosymbiont is highly dependent upon the genetic and cytoplasmic constitution of the host cell; however, only in the case of alpha, which is macronuclear, has a direct association of nucleus and symbiont been demonstrated. The present study developed from a general investigation of the fine structure of nuclear processes in ciliate protozoa; the first Paramecium micronucleus examined showed numerous bacterium-like particles associated with the nuclear envelope. This paper reports the specific association of that symbiont, which structurally resembles Gram-negative bacteria and perhaps rickettsiae, primarily with the micronucleus but also with the macronucleus of Paramecium.

\section{METHODS}

The original culture identified as Paramecium multimicronucleatum was obtained in 1962 from Carolina Biological Supply Company, Elon College, North Carolina, U.S.A. Since that time organisms have been maintained with bacteria in a standard hay + lettuce medium. Paramecia were collected from newly established cultures by micropipette and fixed by exposure to osmium tetroxide vapour as previously described (Jenkins, I964). Following rapid ethanol dehydration, the protozoa were embedded singly in methacrylate which contained $2 \%(\mathrm{v} / \mathrm{v})$ of divinylbenzene as a cross-linking 
agent or an Epon-Araldite mixture according to procedures reported earlier (Jenkins, 1967). Sections were cut with an LKB Ultrotome equipped with glass knives, picked up on grids with Formvar-carbon films, doubly stained with uranyl acetate and lead citrate, and examined with an $\mathrm{RCA}_{3} \mathrm{G}$ electron microscope operated at $100 \mathrm{kV}$ and equipped with $150 \mu \mathrm{m}$. and $30 \mu \mathrm{m}$. condenser and objective apertures respectively.

\section{RESULTS}

Micronuclei of the paramecia were approximately $2.5 \mu \mathrm{m}$. wide in the greatest dimension and were bounded by an envelope consisting of two membranes separated by a less dense space 15 to $20 \mathrm{~nm}$. wide and having numerous pores about $60 \mathrm{~nm}$. in diameter (Pl. I, fig. I ; Pl. 2, fig. 3). All micronuclei thus far examined had, just inside the nuclear envelope and at the apices of the longest dimension, a small bundle of microtubules cut in cross-section (Pl. I, fig. I; Pl. 2, fig. 2). This apparently consistent configuration is reminiscent of the microtubules in the marginal bundle of mammalian blood platelets as studied by Behnke \& Zelander (1967). The macronucleus was very much larger, with an irregular outline, and was enclosed by an envelope similar to that of the micronucleus except that pores were perhaps more numerous and slightly larger (P1. I, fig. I). Nuclear contents were essentially identical to those described for Paramecium by others (Jurand, Beale \& Young, 1962; Ehret \& DeHaller, 1963). In some sections loose bundles of $12 \mathrm{~nm}$. fibres were seen in the central portions of the micronucleus (Pl. I, fig. I); at high magnification a faint periodicity was evident within the bundle. Vivier \& André (I96I) described similar fibrillar structures within the macronucleus of Paramecium caudatum.

The endosymbiont, tentatively named epsilon, occurred only within bulbous distensions (loculi) of the outer membrane of both micro- and macronuclei (PI. I, fig. I), being more numerous per unit surface area for micronuclei. In fact, the macro nuclear envelope was generally devoid of symbionts except in the region near to a micronucleus. The dilated intermembrane space of the loculus was always continuous with that electron-lucid space between the membranes of the nuclear envelope, and arose by way of a $70 \mathrm{~nm}$. wide channel formed by an outward extension of the external membrane of the envelope (Pl. 2, fig. 2). In some instances, continuity between the outer membranes of the envelopes of micronuclei and the macronucleus existed (Pl. 2, fig. 2); such channels might show a dilation which contains an epsilon particle (P1. 2, fig. 2). A loculus might show multiple connexions to the nuclear envelopes and, in some cases, to the rough-surfaced endoplasmic reticulum (Pl. I, fig. I); however, the locular membrane itself was apparently always devoid of ribosomes. The epsilon particle was always separated from its surrounding membrane by an electron-lucid space 75 to $100 \mathrm{~nm}$. wide. Such uniform separation may indicate a capsule which was not preserved by the preparative techniques employed. Only in instances where the division of a symbiont appeared to have occurred recently were two symbionts present within a single loculus.

The symbiont epsilon is best described as a very short rod, sometimes appearing nearly coccoid, approximately $0.35 \mu \mathrm{m}$. in diameter with longer forms reaching $0.7 \mu \mathrm{m}$. in length (all figures). Each particle was limited by a pair of membranes separated by an electron-lucid space (Pl. 2, fig. 3); the outer membrane, space and inner membrane were each about ro $\mathrm{nm}$. in width. The outer membrane had an irregular external 
surface, while the inner membrane, contiguous with the internal content, had a smooth profile. The cytoplasm of epsilon consisted generally of closely packed granules $17 \mathrm{~nm}$. in diameter; in some cases, regions appear to lack these particles and might represent nuclear areas (Pl. 2, fig. 3). The cytoplasm occasionally contained small membranous whorls similar to mesosomes (P1. 2, fig. 3). Deeply constricted forms as shown in Pl. 2, fig 3, were presumably in division, and had a loculus with a concentric indentation. The means by which the two resulting symbionts came to lie within separate loculi has not been observed.

\section{DISCUSSION}

A recent study by Jurand \& Preer (1969) led these workers to conclude that lambda and sigma particles are flagellated bacteria. Earlier, Beale \& Jurand (1966) had shown that three types of mu particles are bacterium-like but did not identify the particles as bacteria. Stevenson (1969) presented biochemical evidence which seemed to establish quite clearly that the mu particle is a symbiotic bacterium. Also, Wagtendonk, Clarke \& Godoy (1963) demonstrated that lambda grown in vitro are Gram-negative rods. The particle epsilon described in this work most resembles Gram-negative bacteria (Glauert, I962; Bladen \& Waters, 1963) or perhaps rickettsia (Ito \& Vinson, 1965; Entwistle, Robertson \& Juniper, 1968) although no pleomorphic forms typical of rickettsia have been observed. The convoluted cytoplasmic invaginations of the plasma membrane described here are not common to Gram-negative bacteria or rickettsia but have been described for both (Lee, I960; Ito \& Vinson, 1965). With only fine structure evidence available it is not possible to identify the exact nature of the symbiont but, in accordance with the conclusions of Beale et al. (1969) reached after an extensive study of endosymbionts, it seems most appropriate to identify epsilon as a bacterium. Endosymbionts of paramecia, with the exception of alpha which is found within the macronucleoplasm (Beale et al. 1969), are cytoplasmic, either embedded directly in the cytoplasmic matrix (for example: mu, Beale \& Jurand, I966) or enclosed either singly or in multiples within membranous vacuoles which are in turn embedded in the cytoplasm as is the case for lambda (Jurand \& Preer, 1969). Sonneborn (1965) stated that mu has a capsular sheath which is in contact with the cytoplasmic matrix. Epsilon is found exclusively within bulbous extensions of the outer membrane of the nuclear envelopes. Such a localization is unique and is thought not to be a transient one since paramecia at different division stages all show the endosymbiont to be nuclear envelope-associated. Although the intracisternal space of the endoplasmic reticulum is continuous with the lumen between membranes of the nuclear envelope, epsilon is not found within the cisternae proper. Also, while extensions of granular endoplasmic reticulum might arise from an occupied loculus, the membrane of the loculus always lacks ribosomes. In this regard, gamma, which is very similar to epsilon in structure and size (Beale et al. 1969), is limited by a vacuole which does bear ribosomes and is thought to be granular endoplasmic reticulum.

The peculiar intracellular location of epsilon seems a most favourable one in terms of communication with both the cytoplasm and the nucleus, at the same time separating the symbiont from possible abrasive cytoplasmic factors. Epsilon is not in direct contact with the nuclear contents; the loculus and the intermembrane space of the nuclear envelope are continuous and are separated from the nucleoplasm by whatever barrier is imposed by the medial components of nuclear pores which are continuous 
through the membranes. Before assuming that the intermembrane location might relate specifically to the advantage of nuclear association, it should be remembered that the lumen of a loculus is often continuous with the intracisternal space of the granular endoplasmic reticulum and could leave the symbiont in direct contact with a product of protein synthesis. The possibility certainly exists that the nuclear association is of benefit to the paramecium by providing ready nuclear access to a product of symbiont synthesis such as purines which are speculated to be a product of endosymbiote metabolism in at least one case (van Wagtendonk et al. 1963). The intermembrane location may not relate directly to nutritional factors alone but also to the mechanism of perpetuation for epsilon. Unfortunately it is not known whether this symbiont shows the same specificity for the genotype of the host that is known to be necessary for the maintenance of other particles (Gibson \& Beale, 196I ; Gibson \& Sonneborn, I964). However, on the basis of the single intracellular location, it seems most probable that epsilon requires nuclear contribution for its existence. Since the nuclear envelopes of both macronuclei and micronuclei remain intact during ciliate division, distribution of epsilon to both daughter cells seems assured and always in association with nuclear material. Whatever the advantage, it appears that epsilon has become adapted to a most unique and favourable intracellular location.

This investigation was supported by NSF Grant GB 6405 .

\section{REFERENCES}

Beale, G. H. \& JuRAND, A. (1960). Structure of the mate-killer (mu) particles in Paramecium aurelia, stock 540. Journal of General Microbiology 23, 243-252.

Beale, G. H. \& JuRAND, A. (1966). Three different types of mate-killer (mu) particles in Paramecium aurelia (syngen I). Journal of Cell Science I, 3I-34.

Beale, G. H., JuRAND, A. \& Preer, J. R. (I969). The classes of endosymbiont of Paramecium aurelia. Journal of Cell Science 5, 65-9I.

BEHNKE, O. \& ZELANDER, T. (1967). Filamentous substructure of microtubules of the marginal bundle of mammalian blood platelets. Journal of Ultrastructure Research 19, 147-165.

BLADEN, H. A. \& WATERS, J. F. (1963). Electron microscopic study of some strains of bacteroides. Journal of Bacteriology 86, I339-I344.

EHRET, G. F. \& DeHAlleR, G. (1963). Origin, development and maturation of organelles and organelle systems of the cell surface of Paramecium. Journal of Ultrastructure Research Suppl. 6, 3-42.

Entwhistle, P. F., Robertson, J.S. \& JuniPeR, B. E. (I968). The ultrastructure of a rickettsia pathogenic to a saturnid moth. Journal of General Microbiology 54, 97-104.

Grbson, I. \& BeAle, G. H. (I96I). Genetic basis of the mate-killer trait in Paramecium aurelia, stock 540. Genetical Research 2, 82-91.

Gibson, I. \& SonNEborn, T. M. (1964). Is the metagon an m-RNA in Paramecium and a virus in Didinum? Proceedings of the National Academy of Sciences of the United States of America 52, $869-876$.

Glauert, A. M. (1962). The fine structure of bacteria. British Medical Bulletin r8, 245-250.

ITo, S. \& Vinson, J. W. (1965). Fine structure of Rickettsia quintana cultivated in vitro and in the louse. Journal of Bacteriology 89, 48I-495.

Jenkins, R. A. (1964). Osmium tetroxide vapour fixation of protozoan cells. Journal of Cell Biology 23, $46 \mathrm{~A}$.

JENKINS, R. A. (1967). Fine structure of division in ciliate protozoa. I. Micronuclear mitosis in Blepharisma. Journal of Cell Biology 34, 463-48r.

Jurand, A., Beale, G. H. \& Young, M. R. (1962). Studies on the macronucleus of Paramecium aurelia. Journal of Protozoology 9, 122-131.

Jurand, A. \& Preer, L. B. (1969). Ultrastructure of flagellated lambda symbionts in Paramecium aurelia. Journal of General Microbiology 54, 359-364. 


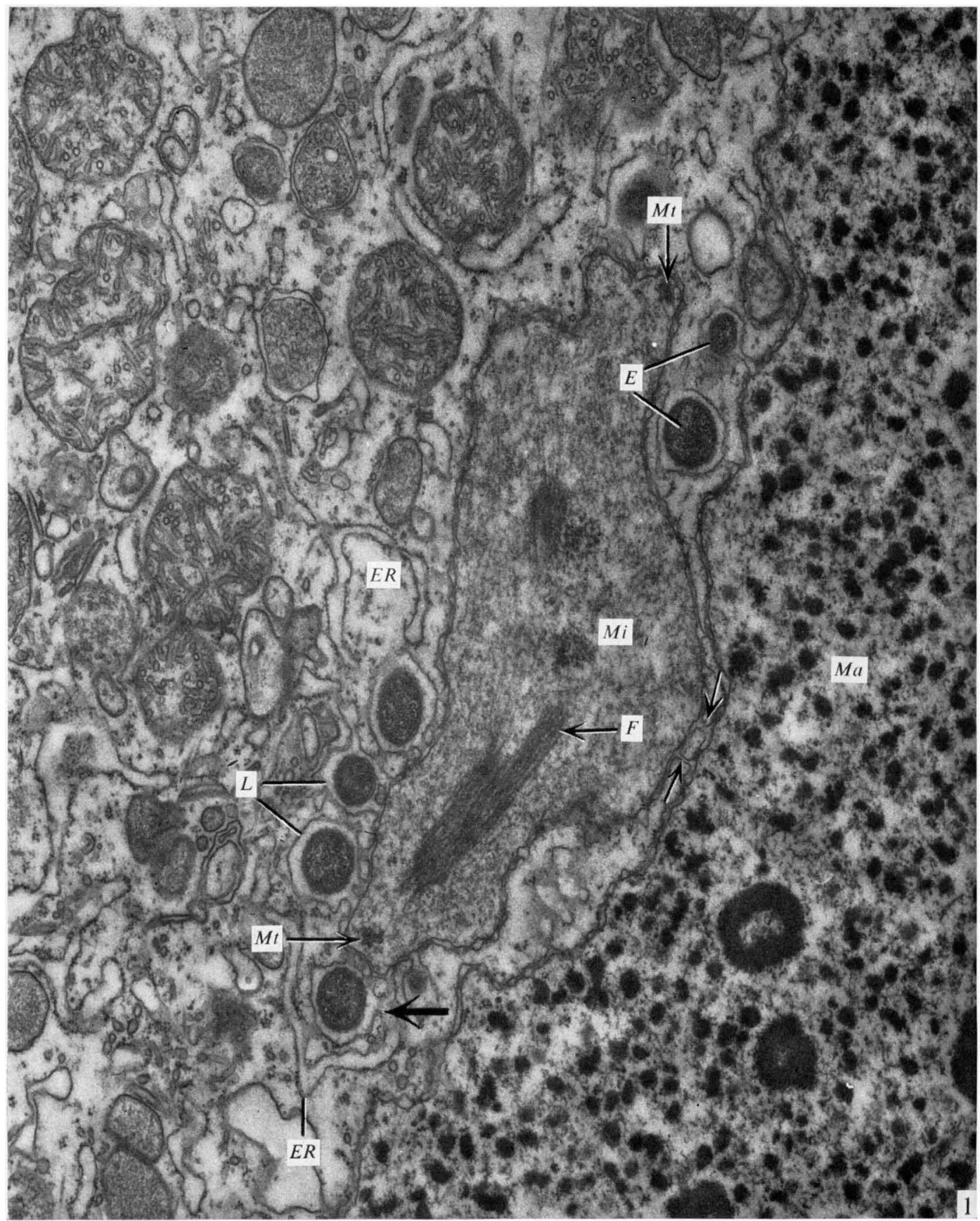


Journal of General Microbiology, Vol. 6I, No. 3

Plate 2
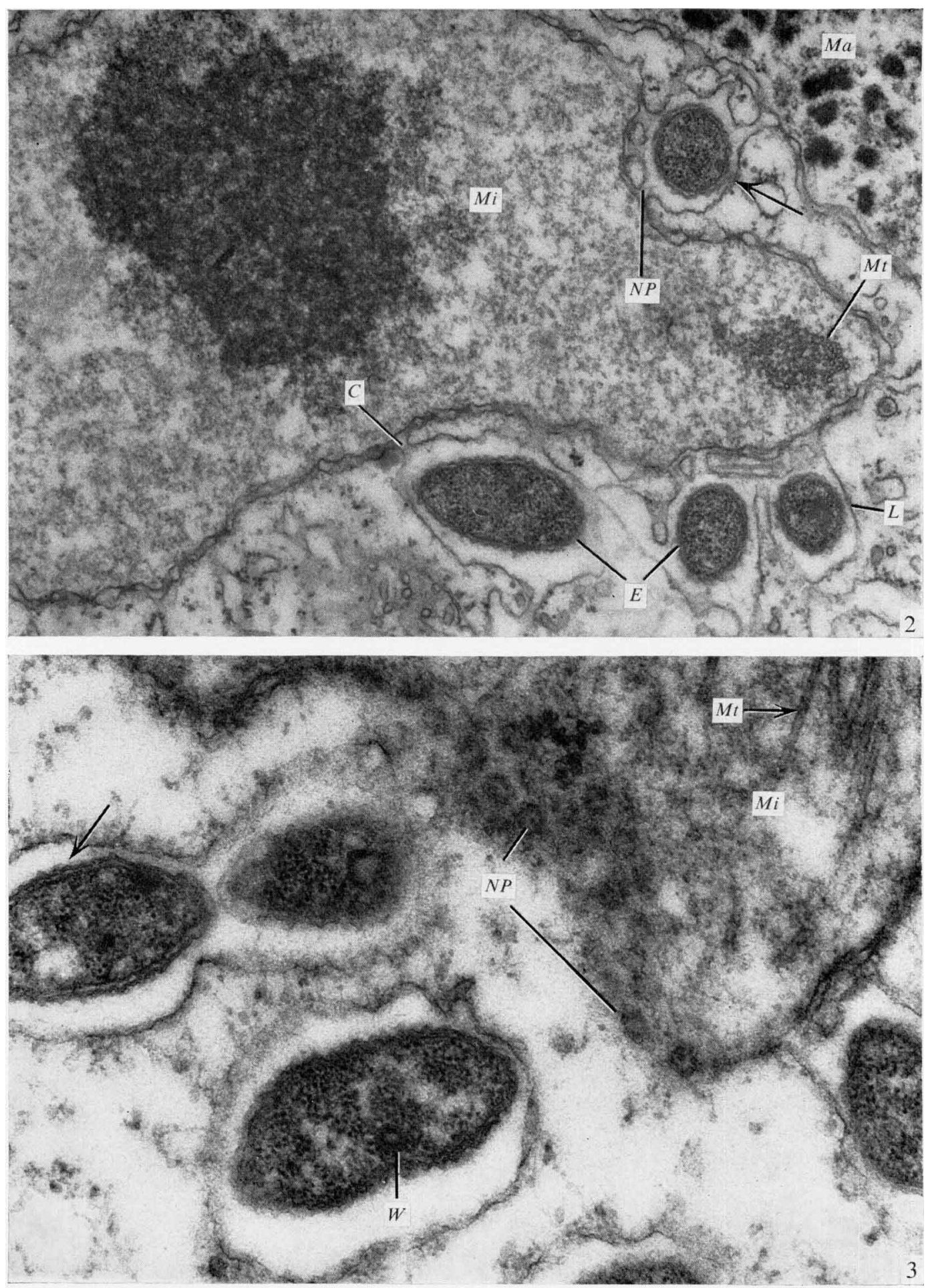

R. A. JENKINS 
LEE, S. (1960). Fixation of Escherichia coli spheroplasts for electron microscopy. Experimental Cell Research 2I, 252-256.

SONNEBORN, T. M. (1965). The metagon: RNA and cytoplasmic inheritance. American Naturalist 99, 279-307.

SteVenson, I. (1969). The biochemical status of $\mu$ particles in Paramecium aurelia. Journal of General Microbiology 57, 61-75.

VIVIER, E. \& ANDRÉ, J. (1961). Existence d'inclusions d'ultrastructure fibrillaire dans le macronucleus de certain souches de Paramecium caudatum Ehr. Comptes Rendus Hebdomadaire des Séances de l'Académie des Sciences, Paris 252, 1848-1850.

van Wagtendonk, W. J., Clarke, J. \& Godoy, G. A. (1963). The biological status of lambda and related particles in Paramecium aurelia. Proceedings of the National Academy of Sciences of the United States of America 50, 835-838.

\section{EXPLANATION OF PLATES}

Symbols: $C=$ channel, $E=$ epsilon particle, $E R=$ endoplasmic reticulum, $F=$ fibres, $L=$ loculus, $M a=$ macronucleus, $M i=$ micronucleus, $M t=$ microtubules, $N P=$ nuclear pore, $W=$ membranous whorl.

PLATE I

Fig. I. A portion of a longitudinal section of Paramecium which shows the macronucleus $(M a)$ and micronucleus $(M i)$ in apposition and with points of contiguity between the outer membranes of their envelopes (arrows). Numerous epsilon particles $(E)$ are present in loculi $(L)$ around the micronucleus. Note the cross-sections of microtubules $(M t)$ at the opposite ends of the micronucleus; all micronuclei so far examined, whether in division or not, show such a disposition of microtubules. The most conspicuous component of the micronucleoplasm is generally the dense, central mass, presumed to be chromatin, as shown in Pl. 2, fig. 2; however, many nuclei are found to have quite extensive bundles of fibres $(F)$. Whether these fibres are present at the expense of the central mass is not clear. Endoplasmic reticulum $(E R)$ shows continuity with the membrane of a loculus at the broad arrow. Mitochondria and other cytoplasmic inclusions are shown at the left side of the figure. $\times 22,000$.

\section{PLATE 2}

Fig. 2. The micronucleus (Mi) of Paramecium occupies the greater amount of the micrograph; epsilon particles $(E)$ are present within loculi $(L)$ which are formed by extensions of the nuclear envelopes via channels $(C)$ initiated at the outer envelope membrane. A loculus with multiple connexions to the micronuclear and macronuclear envelopes occurs at the arrow. Cross-sections of microtubules within the micronucleus occur at $M t$. A small portion of the macronucleus $(M a)$ is shown in the upper right. $\times 33,000$.

Fig. 3. A high-power micrograph showing details of the epsilon particles. The two membranes of epsilon are visible at the arrow. Within a particle, a membrane whorl is discernible at $W$; the particulate cytoplasm and nuclear areas are also visible. Note the indented membrane of theloculus containing two epsilon particles. The envelope of the micronucleus $(M i)$ shows numerous annuli of nuclear pores $(N P)$. Intranuclear microtubules $(M t)$ are present in this dividing micronucleus. $\times 74,000$. 